\title{
Métricas de sumarização automática de texto em tarefas de um Ambiente Virtual de Aprendizagem
}

\author{
Fábio Bif Goularte $^{1}$, Beatriz Wilges ${ }^{2}$, Silvia Modesto Nassar ${ }^{1}$, Renato Cislaghi ${ }^{3}$ \\ ${ }^{1}$ Programa de Pós-Graduação em Ciência da Computação - Universidade Federal de \\ Santa Catarina (UFSC) \\ ${ }^{2}$ Programa de Pós-Graduação em Engenharia e Gestão do Conhecimento - Universidade \\ Federal de Santa Catarina (UFSC) \\ ${ }^{3}$ Programa de Pós-Graduação em Métodos e Gestão em Avaliação - Universidade \\ Federal de Santa Catarina (UFSC) \\ Caixa Postal 88.040-900 - Florianópolis - SC - Brasil \\ \{fabio.bif@posgrad.ufsc.br\}, \{beaw, silvia, cislaghi@inf.ufsc.br\}
}

\begin{abstract}
This paper proposes a method for text summarization to automate part of the correction process of dissertative activities in a Virtual Learning Environment (VLE). To test the method a set (CORPUS) of texts was created. Tests were conducted to evaluate the performance and the impact of summarization. The precision of the method was the best among the compared tools and the impact of summarization in the context of a VLE was considered positive by teachers.
\end{abstract}

Resumo. Este artigo propõe um método de sumarização de texto para automatizar parte do processo de correção das atividades dissertativas de um Ambiente Virtual de Aprendizagem (AVA). Para testar o método foi criado um corpus de textos. Os testes foram conduzidos para avaliar o desempenho e o impacto da sumarização. A precisão do método foi a melhor entre as ferramentas comparadas e o impacto da sumarização no contexto de um AVA foi considerado positivo pelos professores.

\section{Introdução}

Sumarizar é resumir um texto ou conjunto de textos, extraindo as informações mais importantes sem que este perca o sentido do texto original. A sumarização automática caracteriza-se pela geração de um resumo através de métodos computacionais associados a tarefas de Recuperação da Informação (RI) e Mineração de Texto [Aggarwal e Zhai 2012].

A ampla utilização da web e das tecnologias, tais como: dispositivos móveis, redes sociais, sistemas de informação eletrônicos, têm ocasionado um aumento exponencial do volume de informações e dados que são publicados. Segundo Hilbert e López (2011) a quantidade de dados que estão sendo coletados ultrapassou a capacidade humana de armazená-los. O mundo de hoje é fundamentado em informação, sendo que a maior parte está online [Nenkova e Mckeown 2011].

Não obstante, essa mesma tendência é encontrada em Ambientes Virtuais de Aprendizagem (AVA), onde o usuário se depara com o problema clássico de sobrecarga de informação. Segundo Rigo et al. (2013) o crescimento da adoção de modelos de 
Educação a Distância e a necessidade de atendimento em maior escala dos estudantes vem gerando demandas específicas, em especial à atuação de professores e tutores.

$\mathrm{O}$ aumento do volume de material textual presente em AVAs implica em maior tempo para leitura, pesquisa e tratamento. Este cenário tem motivado a pesquisa de novas abordagens para organização automática de parte destas informações [Machado 2010].

Como o fluxo de informações em um texto não é uniforme, o que significa que algumas partes são mais importantes do que outras, este artigo propõe um método genérico de sumarização automática para identificar as principais informações nas atividades dissertativas em AVAs. O método segmenta as atividades em sentenças e as classifica por meio de métricas estatística de determinadas características. Para avaliar este método foram utilizados dados gerados em um AVA, originados das postagens de tarefas realizadas pelos estudantes.

Um sumário pode contribuir para reduzir os esforços de pesquisa e leitura de usuários (tutor ou agente) de AVAs, assim como em contextos onde seja necessário lidar com um grande volume de informação textual. Além disso, um sumário também pode ser utilizado como link para um determinado documento, ou parte de um documento. Outra importante utilidade pode ser a possibilidade de trazer indicadores para uma avaliação baseada em palavras-chave esperadas pelo professor, na descrição da atividade do estudante.

Este artigo está organizado da seguinte forma: a segunda seção apresenta os trabalhos relacionados. A terceira descreve sobre o estado da arte em sumarização automática de texto por extração. Na quarta seção é apresentada a definição do método proposto. Na quinta seção é apresentada a metodologia, os resultados e discussões; por fim, são apresentadas as considerações finais.

\section{Trabalhos relacionados}

O desafio deste trabalho encontra-se não apenas na identificação das informações relevantes das atividades dissertativas de um AVA, mas também que essas informações sirvam de apoio e facilitem o processo de correção, como por exemplo, na avaliação por parte dos docentes ou tutores. A correção de algumas atividades dissertativas requer muito tempo e a disponibilidade da maioria dos educadores é limitada [Ávila; Soares, 2013]. Um sumário pode auxiliar na visão do conteúdo da atividade, pois apresenta um texto informativo e reduzido. Além disso, dificilmente um educador consegue manter o mesmo nível de exigência na correção de um grande volume de atividades.

Pesquisas que avaliam a viabilidade da sumarização automática no contexto de um AVA já foram tema de alguns trabalhos. No trabalho de Ferreira et al. (2009) verificou-se a precisão de sumários produzidos por humanos em relação aos produzido por uma ferramenta, analisando as questões de informatividade e qualidade. Alguns sumários produzidos por ferramentas apresentaram dificuldades à compreensão humana. Ainda assim, os resultados demonstraram um ganho significativo de produtividade com a obtenção de sumários fiéis aos textos originais. A sumarização em determinados locais de um AVA, como os módulos de tarefas dissertativas, é uma alternativa viável e interessante. 
O trabalho de Ávila e Soares (2013) apresentou um estudo que também aborda o problema da sobrecarga de informação em AVAs. Os autores utilizam algoritmos de comparação textual combinados com técnicas de pré-processamento de textos para a correção de avaliações e exercícios dissertativos em AVAs. Através de métricas de similaridade se compara a resposta submetida pelo estudante com a resposta padrão esperada. Uma ferramenta foi desenvolvida para testar e validar essa abordagem.

Outro trabalho que também demonstra que a sumarização automática de texto oferece vantagens no contexto educacional é o de Noorbehbahani e Kardan (2009). Os autores apresentam um método de adaptação de conteúdo textual para cursos em sistemas e-learning, com base na geração de resumos personalizados, segundo os interesses do usuário. O método alcançou 0,57 de correlação entre os escores médios e os resultados foram considerados satisfatórios.

As propostas mencionadas utilizam diferentes técnicas para lidar com o problema de volume de informação em AVAs. Esta proposta se destaca por reunir as seguintes características: (a) apresentar um método que utiliza técnicas de préprocessamento de texto para melhorar o resultado do método de sumarização; (b) descrever e quantificar as métricas que compõem esse método; (c) propor métricas de sumarização de texto para AVAs que são adaptadas da literatura; (d) utilizar dados reais de um AVA para a avaliação; (d) demonstrar a eficiência do método com relação a outras ferramentas de sumarização; (e) desenvolver um método que possa ser utilizado para mais de um estilo de texto.

\section{Sumarização automática de texto}

Existem duas técnicas clássicas de sumarização automática: abstração e extração. A sumarização por abstração utiliza técnicas formais e modelos linguísticos. Para construir um sumário segundo essa técnica, o texto fonte é analisado semanticamente com base na identificação da relação entre as sentenças (frases). O sumário gerado é uma versão reformulada do texto original [Das e Martins 2007]. A sumarização por extração produz um sumário a partir de sentenças selecionadas do texto original, através de métodos estatísticos e/ou empíricos [Balage Filho, Pardo e Nunes, 2007].

O processo de sumarização por extração pode ser dividido em duas etapas: préprocessamento e processamento. A etapa de pré-processamento, geralmente inclui: identificação das sentenças, eliminação de termos que não agregam informações relevantes e obtenção do radical de cada palavra. Na etapa de processamento, as características que influenciam na relevância das sentenças são calculadas e atribui-se um peso, assim as sentenças são classificadas e ranqueadas.

\subsection{Características do texto}

O primeiro passo na sumarização pela técnica de extração é identificar quais são as características textuais, que serão levadas em consideração para definir a importância das sentenças.

Conforme as pesquisas de Das e Martins (2007), Noorbehbahani e Kardan (2009), Gupta e Lehal (2010) e Chandra, Gupta e Paul (2011) algumas características que remetem a relevância de uma sentença e que foram selecionadas para o método proposto são apresentadas. Keywords: geralmente keywords são nomes para coisas. Para determinar as keywords, por exemplo, pode-se utilizar a medida $t f$-idf ou algum 
método que envolva análise morfológica. Partindo do pressuposto de que a ideia principal de um texto pode ser expressa por um conjunto de keywords, a sentença que apresentar keywords têm maior chance de ser incluída no sumário. Título: palavras do título são indicativos do tema do documento, sendo assim, sentenças com palavras do título são mais importantes do que as sentenças onde isso não ocorre. Local da sentença: está característica pode envolver o posicionamento da sentença com relação ao parágrafo, com relação a uma seção do texto, ou ainda, com relação ao documento todo. As primeiras e as últimas sentenças de um parágrafo tendem a ser mais importante, assim como os primeiros e os últimos parágrafos. Comprimento da sentença: sentença muito longa ou curta, geralmente não é incluída no sumário.

Existem outras características estatísticas e linguísticas que podem ser encontradas na literatura e todas são importantes para o processo de sumarização de texto. Porém, o resultado desse processo depende de como elas são exploradas e combinadas e do propósito da aplicação.

As características descritas nessa seção são, historicamente, as mais utilizadas em trabalhos de pesquisa de diversas áreas, inclusive na educação. Isso facilita a avaliação do método.

\section{Estruturação do método}

A estrutura de execução que envolve as etapas do método de sumarização proposto é composta por dois processos chaves: pré-processamento e análise das características. A Figura 1 ilustra a composição dessas etapas, bem como seus respectivos processos.

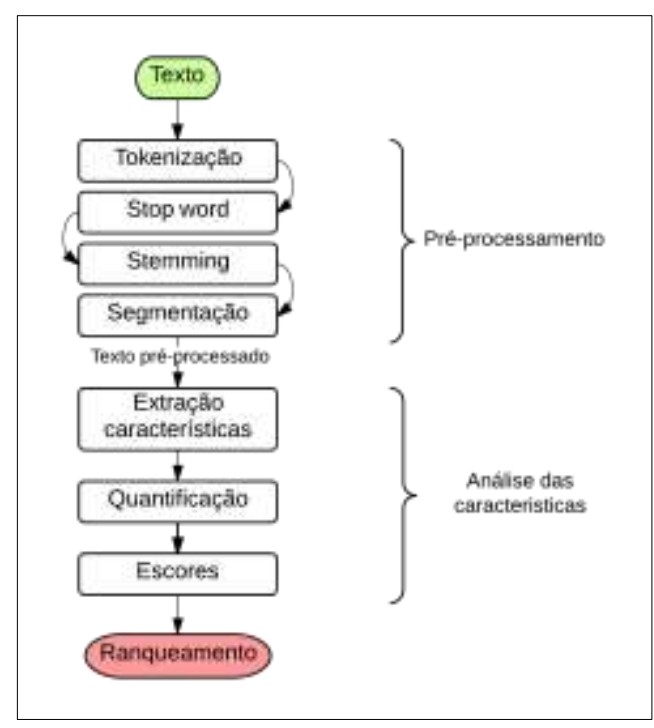

Figura 1. Estrutura do método

Alguns métodos padrões de pré-processamento e mineração de texto são aplicados. A tokenização é a etapa utilizada para decompor o texto de entrada em palavra (tokens) que o compõe. Stop words são palavras de significado irrelevante como, por exemplo, artigo, preposição, advérbios, números, pronomes e pontuação. Stop words são removidas porque não trazem informação semântica sobre as sentenças. Stemming é a etapa utilizada para remover sufixos e prefixos das palavras, reduzindo um termo ao seu radical. A segmentação consiste em delimitar o texto de entrada em 
sentenças. Ao final do pré-processamento, cada sentença estará representada na forma de um vetor de termos.

No processo de análise das características, a extração das caraterísticas é a etapa onde se identifica os critérios das métricas. A quantificação é a etapa de cálculo, que é implementado de forma local e global. Local quando envolvem critérios de localização, quantificação dos termos nas sentenças e no texto como um todo; e global, através da definição da similaridade entre os termos das sentenças. Escores é a etapa de somatórios das características das sentenças e, a etapa de ranqueamento consiste em atribuir pontuação às sentenças e produzir um ranque. O Quadro 1 apresenta as características analisadas.

\section{Quadro 1. Características analisadas}

\begin{tabular}{|c|c|c|c|}
\hline Característica & Métrica & Descrição & Justificativa \\
\hline Localidade (L) & L & $\begin{array}{l}\text { A localização das sentenças no } \\
\text { texto. }\end{array}$ & $\begin{array}{l}\text { As primeiras e as últimas } \\
\text { sentenças do texto tendem a ser } \\
\text { mais importantes. }\end{array}$ \\
\hline Tamanho $(\mathrm{T})$ & $\mathrm{T}$ & O tamanho da sentença. & $\begin{array}{l}\text { A sentença incluída no sumário } \\
\text { não devem ser muito longa ou } \\
\text { curta. }\end{array}$ \\
\hline \multirow{2}{*}{ Palavras-chave $(\mathrm{K})$} & K1 & O número de termos da sentença & $\begin{array}{l}\text { A sentença que apresentar } \\
\text { palavras-chave tem maior chance } \\
\text { de ser incluída no sumário. }\end{array}$ \\
\hline & K2 & $\begin{array}{l}\text { O número de termos do título na } \\
\text { sentença. }\end{array}$ & $\begin{array}{l}\text { Sentenças com palavras do título } \\
\text { são mais importantes do que onde } \\
\text { isso não ocorre. }\end{array}$ \\
\hline
\end{tabular}

\section{Procedimentos metodológicos}

Está seção apresenta a descrição detalhada das métricas relacionadas a cada uma das características apresentadas no Quadro 1, a base de dados e o AVA utilizados, a atividade selecionada para a construção do corpus de textos e as medidas de avaliação.

\subsection{Métrica da localização (L)}

A maneira padrão de ranquear as sentenças conforme sua posição no parágrafo não se demonstrou adequada para este trabalho. Muitas tarefas apresentavam parágrafos com apenas uma ou duas sentenças. Ocorreu que várias sentenças recebiam um peso que não estava de acordo com a justificativa encontrada na literatura para está métrica [Gupta e Lehal 2010] [Chandra, Gupta e Paul 2011]. Devido ao motivo mencionado fez-se necessário adaptar a medida de localidade. A medida $L$ atribui maior relevância as primeiras e as últimas sentenças do texto, a partir da região central.

Para encontrar a localização das sentenças no texto, conforme sua importância, o cálculo $L$ é definido pela razão entre a posição de cada sentença $p_{i y}$ e o número de sentenças $n_{s y}$ no conjunto $B_{y}$.

$$
L\left(p_{i y}, n_{S y}\right)=\left\{\begin{array}{ll}
1-\left(\frac{p_{i y}-1}{n_{S y}}\right) & ; \text { Se } y=1 \\
\frac{p_{i y}}{n_{S y}} & ; \text { Se } y=2
\end{array} \quad \operatorname{com} p_{i y}=1, \ldots, n_{S y}\right.
$$

Onde $L$ é um valor normalizado entre 0 e 1 . A sentença mais significativa terá $L=1$, enquanto que a última terá $L$ próximo a 0 . Sendo $S$ o conjunto das sentenças $S$ do texto, têm-se os subconjuntos $B_{1}$ e $B_{2}$, os quais são analisados individualmente. 


\subsection{Métrica do tamanho $(T)$}

Uma forma de calcular a relevância de uma sentença em relação ao seu tamanho é a razão entre a quantidade de termos pela quantidade de termos da maior sentença do texto [Das e Martins 2007]. Dependendo da estruturação do texto, sentenças demasiadamente longas podem ser privilegiadas. Esse fato motivou a formulação da métrica $T$ que é definida da seguinte forma:

$$
T=\ln \left(\bar{t}_{s y}-\left|\frac{\bar{t}_{s y}-t_{s i y}}{\sigma_{y}}\right|\right)
$$

O valor de $T$ é calculado pelo logaritmo natural da média da quantidade dos termos $\bar{t}_{s y}$ de $B_{y}$, menos o valor absoluto do escore padrão. Onde $t_{s i y}$ é a média da quantidade dos termos da sentença observada e $\sigma_{y}$ é o desvio padrão. $O$ peso que resulta da medida $T$ aumenta conforme a quantidade de termos da sentença se aproxima da média $\bar{t}_{s y}$.

\subsection{Métricas de palavras-chave (K1 e K2)}

As métricas $K 1$ e $K 2$ se referem a característica das palavras-chave que são definidas por critérios de similaridade. Um dos critérios de similaridade mais utilizados em tarefas de RI é a medida $t f$-idf, e com base nela, a medida $t f$-isf (frequência do termo - frequência inversa da sentença) calcula o valor médio da frequência de todos os termos do texto.

$$
w_{i j}=t f_{i j} \times i s f=\frac{f_{i j}}{\sum_{k=1}^{N} f_{k j}} \times \log \frac{N}{\left|n_{i}\right|}
$$

O peso $w_{i j}$ é dado pela frequência $t f_{i j}$ multiplicada pela medida de importância geral do termo isf. A $f_{i j}$ é a quantidade de ocorrências do termo $t_{i}$ no documento $d_{j}$ e $k$ é a quantidade de termos distintos. A frequência é normalizada pela isf para prevenir um viés em documentos longos ou quando se lida com mais de um documento. A isf é definida como sendo o logaritmo do quociente entre o número total de sentenças do documento $N$ e o número de sentenças que contém o termo $n_{i}$.

O número de vezes que um termo aparece no documento aumenta proporcionalmente o seu peso, sendo inversamente proporcional a frequência do termo no documento. Segundo Baeza-Yates e Ribeiro-Neto (2011) essa afirmação é baseada na observação de que a alta frequência dos termos é importante para descrever documentos. Dessa forma, define-se um escore à sentença, denominado $K 1$.

$$
K 1=\frac{\sum_{i=1}^{k} w_{i j}\left(s_{i}\right)}{\operatorname{Max}\left(\sum_{i=1}^{k} w_{i j}\left(s_{i}^{N}\right)\right)}
$$

O escore $K 1$ é definido pela razão entre o somatório dos pesos dos termos $w_{i j}$ da sentença $s_{i}$ e o maior somatório dos pesos dos termos em todas as sentenças $s_{i}^{N}$.

A medida $K 2$ pontua uma sentença conforme as palavras que se assemelham ao título ou subtítulo. Cada termo do título é consultado por sentença, estabelecendo assim um modelo de espaço vetorial ponderado.

$$
K 2=\operatorname{sim}\left(s_{i}, q\right)=\sum_{j=1}^{k} w_{i j} w_{y j}
$$

Onde $s_{i}$ é a i-ésima sentença, $q$ é a consulta do título, $w_{i j}$ é o peso do termo da sentença e $w_{y j}$ é o peso do termo do título. Todas as métricas apresentadas são normalizadas para melhorar o processo de análise e avaliação. 
Ao final do processo a sentença apresenta um escore que é o somatório de todas as métricas, normalizado pelo maior valor.

$$
\operatorname{score}\left(s_{i}\right)=\frac{\sum_{i=1}^{k} L T K 1 K 2\left(s_{i}\right)}{\operatorname{Max}\left(\sum_{i=1}^{k} L T K 1 K 2\left(s_{i}\right)\right)}
$$

\subsection{Origem dos dados}

Os dados desta pesquisa são provenientes de um AVA que é utilizado no ensino semipresencial na disciplina de Estatística pelos estudantes de graduação em Engenharia da Universidade Federal de Santa Catarina. Este AVA é baseado na aprendizagem significativa onde o estudante tem a possibilidade de trabalhar os dados de sua pesquisa em diferentes módulos de aprendizagem.

O primeiro passo foi descobrir qual das atividades apresentava maior potencial à sumarização. A Figura 2 ilustra a tarefa dissertativa Correção e Planejamento da Pesquisa (CPP), com maior frequência no AVA, conforme os cursos e disciplinas.

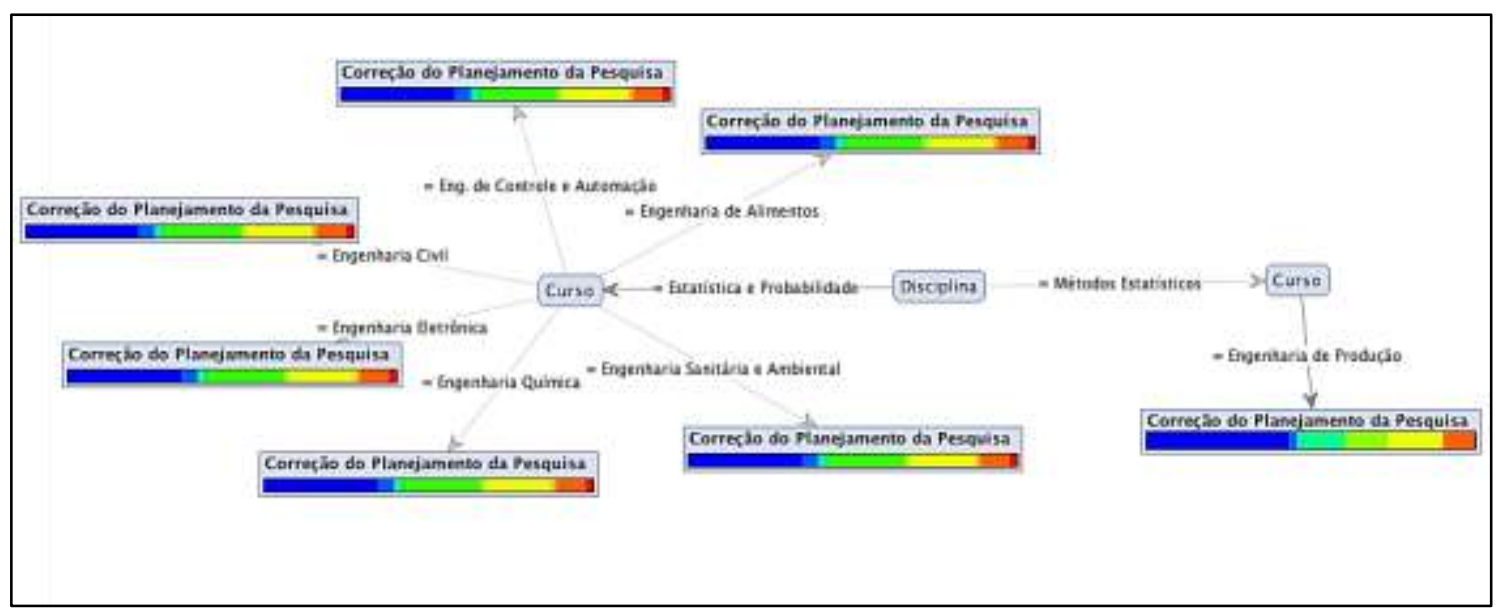

Figura 2. Tarefa mais frequente no AVA independente do curso

A base de dados do AVA armazenava 345 resultados para a tarefa CPP. Algumas tarefas continham texto duplicado ou fora de contexto. Os dados foram filtrados e os itens duplicados foram removidos, restando 122 resultados válidos. Para compor um corpus para a pesquisa, 67 textos da tarefa CPP foram selecionados aleatoriamente por amostragem, ao nível de significância de $95 \%$, com margem de erro de $8 \%$. O corpus totaliza 39.710 palavras, com uma média de 592 palavras por texto e desvio padrão de 358 .

A tarefa CPP não apresentava título ou subtítulos e, sabendo-se que a métrica $K 2$ é o resultado da similaridade das sentenças com relação ao título. $O$ passo seguinte foi aplicar o processamento de linguagem natural na resposta padrão da tarefa para identificar as principais palavras-chave. Palavras-chave indicam o assunto do texto e o mesmo acontece com palavras do título. $\mathrm{O}$ processamento do texto padrão foi realizado na ferramenta Nltk 3.0. O resultado estatístico e linguístico foi analisado pelo professor da disciplina que apontou a relevância para onze termos mais frequentes: variáveis, amostra, censo, nível, significância, amostragem, tamanho, associação, representativa, quantitativa (qt), qualitativa (ql).

O professor da disciplina também assumiu o papel de sumarizador. Foi solicitado ao professor que marcasse quais as partes mais importantes do texto de cada 
tarefa a fim de identificar as sentenças do sumário ideal. O limite máximo adotado para a sumarização humana: um terço do total de linhas do texto original [Ferreira et al., 2009]. Os sumários humanos são utilizados na etapa de avaliação do método onde foram comparados com os sumários produzidos automaticamente.

\subsection{Avaliação do método}

Foram realizados dois tipos de avaliação. O primeiro avaliou a qualidade do método proposto com as medidas clássicas de precisão, cobertura e medida-f. O segundo verificou o impacto da sumarização na tarefa selecionada.

A precisão é medida pela razão entre o número de sentenças relevantes recuperadas e referentes ao sumário ideal, e o número total de sentenças recuperadas. Já a cobertura é definida pela relação entre o número de sentenças relevantes recuperadas e referentes ao sumário ideal, e o número total de sentenças contidas nesse sumário. A medida-f é uma medida geral de desempenho obtida a partir da média harmônica ponderada da precisão e cobertura.

O corpus foi sumarizado pelas ferramentas Gistsumm, Summarize Thingiee (ST), Microsoft Word (MW) e pela implementação do método proposto.

\section{Resultados}

Os sumários automáticos gerados em cada uma das ferramentas foram comparados aos sumários ideais. Os resultados são as médias segundo as medidas de precisão, cobertura e medida-f que são mostradas na Tabela 1 e Figura 3.

Tabela 1. Resultados da avaliação

\begin{tabular}{lccc}
\hline Ferramentas & \multicolumn{3}{c}{ Métricas } \\
\cline { 2 - 4 } $\begin{array}{l}\text { Método } \\
\text { proposto }\end{array}$ & $\mathbf{0 , 6 8 3}$ & 0,695 & $\mathbf{0 , 6 8 8}$ \\
Gistsumm & 0,622 & $\mathbf{0 , 6 9 7}$ & 0,657 \\
ST & 0,564 & 0,550 & 0,558 \\
MW & 0,533 & 0,538 & 0,535 \\
\hline
\end{tabular}

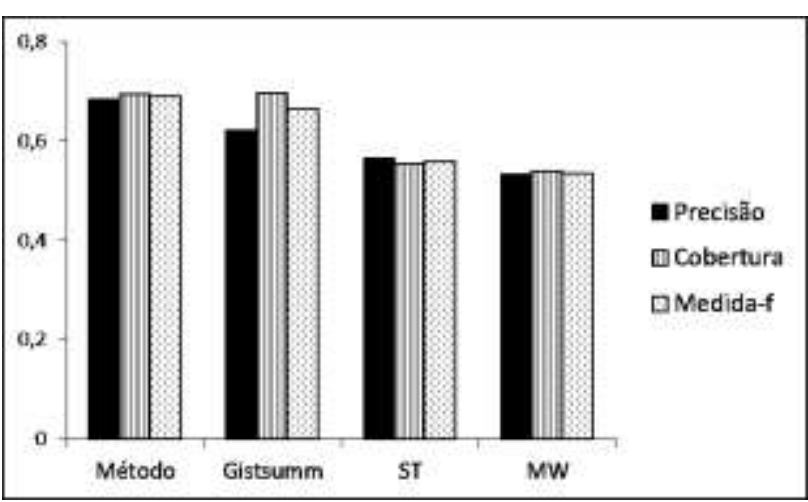

Figura 3. Gráfico dos resultados

Os valores da Tabela 1 variam entre 0 e 1 , onde 0 indica que o sumário automático é diferente do sumário ideal e 1 indica a proximidade máxima entre ambos. Entre as ferramentas analisadas, o método proposto apresentou o melhor índice de precisão média, 0,68. Enquanto que o melhor desempenho com relação a cobertura foi obtido pelo Gistsumm, 0,69. O Gistsumm é uma ferramenta que vem sendo aprimorada, principalmente por pesquisadores brasileiros e apresenta melhores resultados com textos estruturados [Filho, Pardo e Nunes, 2007]. Devido a tarefa CPP não apresentar uma estruturação textual bem definida, a medida de desempenho geral do método proposto obteve o melhor resultado, com um valor de 0,68.

Estes índices tendem a subir de acordo com o aumento do número da amostra. Outro fato que pode influenciar na melhora dos resultados é com relação ao tamanho do texto da tarefa. Conforme mencionado, o desvio padrão de 358 da média de palavras por 
textos pode ser considerado alto. Se a tarefa tem uma quantidade pequena ou grande de palavras em relação a média, considerando que apenas $1 / 3$ do texto fará parte do sumário ideal, os valores de precisão e cobertura acabam sendo piores. Para se melhorar o método, pode-se estabelecer um filtro sobre a quantidade mínima e máxima de palavras que a tarefa deve conter, ou ainda, analisar outras métricas além das utilizadas nesta pesquisa.

O impacto da sumarização em uma tarefa dissertativa de um AVA foi avaliado por meio de um questionário. O questionário foi criado e aplicado em um grupo de sete professores da área de Estatística. Os professores tiveram acesso às tarefas de CPP e a descrição padrão da tarefa (elaborada pelo professor da disciplina), ambas no AVA. Cada professor analisou em média nove tarefas e seus respectivos sumários produzidos pelo método proposto e, então responderam o questionário. As perguntas e as possibilidades de respostas, assim como os escores das possibilidades (5 para ótimo, 4 para bom, 3 para regular, 2 para ruim e 1 para péssimo) são apresentadas na Tabela 2.

Tabela 2. Questionário sobre o impacto da sumarização

\begin{tabular}{|c|c|c|c|c|c|c|c|}
\hline \multirow{2}{*}{\multicolumn{2}{|c|}{ Pergunta }} & \multicolumn{5}{|c|}{ Porcentagem (\%) } & \multirow[b]{2}{*}{ Escore } \\
\hline & & $\begin{array}{c}\text { Ótimo } \\
\text { (5) }\end{array}$ & $\begin{array}{c}\text { Bom } \\
(4)\end{array}$ & $\begin{array}{c}\text { Regular } \\
(3)\end{array}$ & $\begin{array}{c}\text { Ruim } \\
(2)\end{array}$ & $\begin{array}{l}\text { Péssimo } \\
\text { (1) }\end{array}$ & \\
\hline 1 & Como você definiria a qualidade dos sumários? & - & 57,1 & 28,6 & 14,3 & - & 3,4 \\
\hline 2 & Qual o nível de informatividade dos sumários? & 14,3 & 42,9 & 28,6 & 14,3 & - & 3,6 \\
\hline 3 & $\begin{array}{l}\text { Se o método fosse implementado em um AVA, como o } \\
\text { utilizado nesta pesquisa, você acredita que sumarizar } \\
\text { tarefas dissertativas auxilie o professor na avaliação? }\end{array}$ & 28,6 & 28,6 & 28,6 & 14,3 & - & 3,7 \\
\hline 4 & $\begin{array}{l}\text { Qual o nível de coerência textual entre as sentenças } \\
\text { do sumário? }\end{array}$ & - & 28,6 & 28,6 & 28,6 & 14,3 & 2,7 \\
\hline 5 & $\begin{array}{l}\text { As palavras-chave utilizadas para identificar os } \\
\text { principais conceitos da tarefa estavam presentes nos } \\
\text { resumos? }\end{array}$ & 14,3 & 28,6 & 42,9 & 14,3 & - & 3,4 \\
\hline
\end{tabular}

A avaliação do impacto da sumarização em tarefas dissertativas de um AVA foi positiva, com uma média de escore de 3,4 pontos. A informatividade que é o quanto um sumário é fiel a ideia principal do texto original obteve um escore de 3,6. A coerência textual está entre regular e ruim, pois apresentou o escore de 2,7. A coerência textual pode ser melhorada com a adição de outras métricas ao método proposto. A questão 3 obteve o maior escore, com 3,7 pontos - os professores consideraram de regular a bom a possibilidade de contar com a implementação do método proposto em um AVA.

Portanto, a avaliação do método proposto demonstra que as métricas mais comuns de sumarização de texto selecionadas, assim como a métrica $T$ que foi criada, atenderam ao propósito de um método genérico de sumarização automática de texto. O desempenho do método também está sendo avaliado em outro corpus de texto e, ainda que preliminares, os resultados vêm ao encontro dos apresentados nesta pesquisa.

\section{Considerações}

De uma maneira geral, a proposição de atividades dissertativas de aprendizagem é limitada devido ao esforço necessário para corrigi-las. Porém, essa correção pode ser facilitada de posse de um sumário da atividade.

Um sumário deve apresentar conceitos chaves esperados pelo professor na atividade, e estes podem ser recuperados automaticamente por métodos de sumarização. Este trabalho apresenta um método que alia métricas de sumarização de texto e técnicas de pré-processamento. Os resultados demonstram que o método proposto é capaz de sumarizar atividades dissertativas realizadas pelos estudantes. 
A implementação do método proposto em um AVA, pode automatizar uma parte do processo de correção de atividades dissertativas, inclusive em tempo real, pois também há a possibilidade de feedback. Por exemplo, o professor cadastra uma resposta padrão com os conceitos chaves da atividade. Um método automatizado pode comparar a resposta padrão à resposta do estudante, que durante a execução e submissão da atividade pode identificar pontos onde o texto deve ser melhorado. Um agente pedagógico pode ser inserido nessa situação, exibindo, caso seja necessário, dicas para ajudar o estudante a compreender melhor os conceitos da atividade.

\section{Referências}

Aggarwal, C.; Zhai, C. Mining text data. [s.1.] Springer US, 2012. v. 4p. 889-903.

Chandra, M.; Gupta, V.; Paul, S. A statistical approach for automatic text summarization by extraction. International Conference on Communication Systems and Network Technologies (CSNT), p. 268-271, jun. 2011.

Das, D.; Martins, A. F. T. A Survey on Automatic Text Summarization. Literature Survey for the Language and Statistics II course at CMU, v. 4, p. 1-31, 2007.

Ávila, Ricardo L.F de.; Soares, J.M. Uso de técnicas de pré-processamento textual e algoritmos de comparação como suporte à correção de questões dissertativas: experimentos, análises e contribuições. In: SBIE, 2013, Campinas, SP. Anais do $24^{\circ}$ SBIE, 2013.

Ferreira, Fernanda de Melo; Rocha, Hemilis Joyse Barbosa; Júnior, Gilberto Pedro da Silva; Bittencourt, Ig Ibert; Costa, Evandro de Barros . Sumarização de Texto em Ambientes Educacionais na Web. In: SBIE, 2009, Florianópolis, SC. Anais do XX SBIE, 2009.

Balage Filho; Pardo, Thiago Alexandre Salgueiro; Nunes, Maria das Graças Volpe. Sumarização automática de textos científicos: Estudo de caso com o sistema gistsumm. ICMC-USP, 2007.

Gupta, V.; Lehal, G. A Survey of Text Summarization Extractive Techniques. Journal of Emerging Technologies in Web Intelligence, v. 2, n. 3, p. 258-268, 20 ago. 2010.

Hilbert, M.; López, P. The world's technological capacity to store, communicate, and compute information. Science (New York, N.Y.), v. 332, n. 6025, p. 60-5, 1 abr. 2011.

Machado, A.P.; Ferreira, Rafael; Bittencourt, Ig Ibert; Elias, Endhe; Brito, Patrick; Costa, Evandro. Mineração de texto em Redes Sociais aplicada à Educação a Distância. Colabor@-A Revista Digital da CVA-RICESU, v. 6, n. 23, 2010.

Nenkova, A.; Mckeown, K. Automatic Summarization. Foundations and Trends® in Information Retrieval, v. 5, n. 3, p. 235-422, 2011.

Noorbehbahani, Fakhroddin; Kardan, Ahmad. Automated Personalized Summary Generation for Text-Based Courses in e-Learning Systems. In: 4th International Conference on E-Learning: University of Toronto, Canada, 16-17 July 2009. Academic Conferences Limited, 2009. p. 386.

Rigo, Sandro J. et al. O papel do Processamento de Língua Natural e da Representação de Conhecimento na extração de informações em mensagens textuais na Educação a Distância. In: SBIE, 2013, Campinas, SP. Anais do $24^{\circ}$ SBIE, 2013. 